PROCEEDINGS OF THE

AMERICAN MATHEMATICAL SOCIETY

Volume 130, Number 11, Pages 3185-3191

S 0002-9939(02)06481-X

Article electronically published on March 25, 2002

\title{
P.I. ENVELOPES OF CLASSICAL SIMPLE LIE SUPERALGEBRAS
}

\author{
IAN M. MUSSON
}

(Communicated by Lance W. Small)

\begin{abstract}
Let $\mathfrak{g}$ be a classical simple Lie superalgebra. We describe the prime ideals $P$ in the enveloping algebra $U(\mathfrak{g})$ such that $U(\mathfrak{g}) / P$ satisfies a polynomial identity. If the factor algebra $U(\mathfrak{g}) / P$ is not artinian, then it is an order in a matrix algebra over $K(z)$.
\end{abstract}

Throughout this paper we work over an algebraically closed field $K$ of characteristic zero. All unadorned tensor products are taken over $K$. Let $\mathfrak{g}$ be a finite dimensional classical simple Lie superalgebra over $K$. A factor algebra of the enveloping algebra $U(\mathfrak{g})$ satisfying a polynomial identity is called a P.I. envelope of $\mathfrak{g}$. Our aim is to describe all prime P.I. envelopes of $\mathfrak{g}$. If $\mathfrak{g}$ has a nonartinian prime P.I. envelope it is not hard to show that the center of $\mathfrak{g}_{0}$ must be nonzero (Lemma 1.3). Thus by the classification theorem in [K1], $\mathfrak{g}=s \ell(m, n)$ with $m>n \geq 1$ or $\mathfrak{g}=\operatorname{osp}(2,2 n)$.

It was shown by Bahturin and Montgomery that when $\mathfrak{g}=s \ell(m, n)$ with $m>$ $n \geq 1, \mathfrak{g}$ has a nonartinian P.I. envelope. In fact the proof of [BM], Theorem $4.2]$ shows that this is true also when $\mathfrak{g}=\operatorname{osp}(2,2 n)$ although these algebras are omitted from the statement of [BM], Theorems 1.5 and 4.2]. If $\mathfrak{g}=s \ell(m, n)$ with $m>n \geq 1$ or $\mathfrak{g}=\operatorname{osp}(2,2 n)$, then $\mathfrak{g}_{0}=\left[\mathfrak{g}_{0}, \mathfrak{g}_{0}\right] \oplus K z$ where $z$ is central in $\mathfrak{g}_{0}$. Furthermore as a $\mathfrak{g}_{0}$-module via the adjoint action, $\mathfrak{g}_{1}=\mathfrak{g}_{1}^{+} \oplus \mathfrak{g}_{1}^{-}$, a direct sum of two simple submodules. If $\mathfrak{p}=\mathfrak{g}_{0} \oplus \mathfrak{g}_{1}^{+}$, then $U\left(\mathfrak{g}_{0}\right)$ is a homomorphic image of $U(\mathfrak{p})$ and thus any $U\left(\mathfrak{g}_{0}\right)$-module can be regarded as a $U(\mathfrak{p})$-module. Choose a Cartan subalgebra $\mathfrak{h}$ and a system of simple roots for $\mathfrak{g}_{0}$. Let $P^{+}$denote the corresponding set of dominant integral weights. If $\lambda \in P^{+}$let $L_{\lambda}$ be the finite dimensional simple $\left[\mathfrak{g}_{0}, \mathfrak{g}_{0}\right]$-module with highest weight $\lambda$ and set

$$
V(\lambda)=U(\mathfrak{g}) \otimes_{U(\mathfrak{p})}\left(L_{\lambda} \otimes K[z]\right) .
$$

Our main result is as follows.

Main Theorem. Set $P_{\lambda}=\operatorname{ann}_{U(\mathfrak{g})} V(\lambda), n=\operatorname{dim}_{K} L_{\lambda}$ and $N=n 2^{\operatorname{dim} \mathfrak{g}_{1}^{-}}$. Then $P_{\lambda}$ is a prime ideal of $U(\mathfrak{g})$ such that $U(\mathfrak{g}) / P_{\lambda}$ is a subring of the matrix algebra $M_{N}(K[z])$ with Goldie quotient ring $M_{N}(K(z))$. In particular $U(\mathfrak{g}) / P_{\lambda}$ is a prime P.I. algebra.

Conversely, if $P$ is a prime ideal in $U(\mathfrak{g})$ such that $U(\mathfrak{g}) / P$ satisfies a polynomial identity, then $P=P_{\lambda}$ for a unique $\lambda \in P^{+}$.

Received by the editors June 12, 2001.

2000 Mathematics Subject Classification. Primary 17B20, 17 B35.

(C)2002 American Mathematical Society 
If $\mathcal{C}=K[z] \backslash\{0\}$, then $\mathcal{C}$ is an Ore set of regular elements in $U(\mathfrak{g})$. A key step in the proof that $P_{\lambda}$ is prime is to show that the localized module $V(\lambda)_{\mathcal{C}}$ is in a natural way a $U(\mathfrak{g})-F$ bimodule where $F=K(z)$, and then a simple Kac module over $U(\mathfrak{g}) \otimes F$. For the converse we use some results of E.S. Letzter concerning prime ideals in finite extensions of Noetherian rings.

1.1. Let $R$ and $S$ be prime Noetherian rings. An $R-S$ bimodule $M$ is a bond from $R$ to $S$ if $M$ is finitely generated and torsionfree both as a left $R$-module and as a right $S$-module.

Lemma. Suppose $M$ is a bond from $R$ to $S$. Then:

(a) $R$ is artinian if and only if $S$ is artinian.

(b) $R$ is a P.I. ring if and only if $S$ is a P.I. ring.

Proof. (a) This is [J] Theorem 5.2.9].

(b) This is Remark (2) after [BS, Prop. 2.5]. We give some details for the convenience of the reader. Let $D=$ Fract $R$ and $E=$ Fract $S$. By [BS, Prop. 2.5] there exists an integer $t$ such that $D$ embeds in the ring of $t \times t$ matrices $M_{t}(E)$ and $E$ embeds in $M_{t}(D)$. Thus if $S$ is P.I., then $R$ embeds in $M_{t}(E)$ which is a central simple algebra by Posner's Theorem [McR, Theorem 13.6.5], so $R$ is P.I. by the Amitsur-Levitzki Theorem [McR, Corollary 13.3.5]. Similarly if $R$ is P.I. so is $S$.

1.2. Until the end of section 1.4 suppose that $R \subseteq S$ is an extension of Noetherian $K$-algebras of finite Gel'fand-Kirillov dimension. Assume that $S$ is finitely generated and free as a right $R$-module. The following definitions are due to Letzter [L2], [L3].

i) Suppose $P$ is a prime ideal of $S$ and set $B=\operatorname{Fract}(S / P)$. Let $V_{P}$ be the set of prime ideals of $R$ which are right annihilators of simple $B-R$ factor bimodules of $B$.

ii) Suppose $Q$ is a prime ideal of $R$ and set $A=\operatorname{Fract}(R / Q)$. Let $W_{Q}$ be the set of prime ideals of $S$ which are left annihilators of simple $S-A$ factor bimodules of $S \otimes_{R} A$.

In addition set $J_{Q}=\ell-\operatorname{ann}(S / S Q)$ and

$$
X_{Q}=\left\{P \in \operatorname{Spec} R \mid P \text { is minimal over } J_{Q}\right\} .
$$

These definitions are related by the following results.

Theorem. (a) If $Q \in \operatorname{SpecR}$ and $P \in \operatorname{SpecS}$, then

$$
Q \in V_{P} \text { if and only if } P \in W_{Q} .
$$

Furthermore if this condition holds there is a bond from $S / P$ to $R / Q$.

(b) $W_{Q} \subseteq X_{Q}$.

Proof. (a) follows from [L2, Lemma 3.2] and [L1, Lemma 1.1], while (b) follows from the proof of [L2, Proposition 4.2].

1.3. Lemma. Let $\mathfrak{g}$ be a classical simple Lie superalgebra such that there is a prime ideal $P$ in $U(\mathfrak{g})$ with $U(\mathfrak{g}) / P$ a nonartinian P.I. algebra. Then the center of $\mathfrak{g}_{0}$ is nonzero. 
Proof. We apply the results of the two previous subsections, with $R=U\left(\mathfrak{g}_{0}\right)$ and $S=U(\mathfrak{g})$. Choose $Q \in V_{P}$. Then there is a bond from $S / P$ to $R / Q$ by Theorem 1.2. Hence by Lemma $1.1, R / Q$ is a non-artinian P.I. algebra. The result follows from a result of Bahturin; see [BM, page 2837].

1.4. If the equivalent conditions of Theorem 1.2(a) hold we say that $P$ lies directly over $Q$. Recall that a module over a prime Noetherian ring is fully faithful if every nonzero submodule is faithful. We require another result of Letzter L3, Lemma 2.6 (iv)].

Lemma. Suppose that $P$ lies directly over $Q$ and that $M$ is a fully faithful $S / P$ module. Then there exists an $R$-submodule $N$ of $M$ such that $Q=a n_{R} N$ and $N$ is a fully faithful $R / Q$-module.

1.5. Again suppose that $\mathfrak{g}$ is classical simple. We often write $U$ for $U(\mathfrak{g})$. The simple artinian factor rings of $U$ correspond to the finite dimensional simple $\mathfrak{g}$ modules and these have been classified [K1, Theorem 8].

For the remainder of this paper we assume therefore that $P$ is a prime ideal of $U$, such that $U / P$ is a nonartinian P.I. algebra. By Lemma 1.3 this means that $\mathfrak{g}_{0}$ has a nonzero center.

As noted in the introduction we have $\mathfrak{g}_{0}=\left[\mathfrak{g}_{0}, \mathfrak{g}_{0}\right] \oplus K z$, and we can choose $z$ in such a way that $[z, x]= \pm x$ for all $x \in \mathfrak{g}^{ \pm}$. Set $\mathfrak{h}^{\prime}=\mathfrak{h} \oplus K z$, so that $\mathfrak{h}^{\prime}$ is a Cartan subalgebra of $\mathfrak{g}_{0}$ and $\mathfrak{g}$. Fix a non-degenerate invariant bilinear form (, ) on $\left(\mathfrak{h}^{\prime}\right)^{*}$. For $\alpha \in\left(\mathfrak{h}^{\prime}\right)^{*}$, we write $\mathfrak{g}^{\alpha}$ for the corresponding root space. There is a unique $h_{\alpha} \in \mathfrak{h}^{\prime}$ such that $(\mu, \alpha)=\mu\left(h_{\alpha}\right)$ for all $\mu \in\left(\mathfrak{h}^{\prime}\right)^{*}$. Let $\Delta_{1}^{+}$be the set of roots of $\mathfrak{g}_{1}^{+}$. For $\alpha \in \Delta_{1}^{+}$, choose $e_{ \pm \alpha}$ such that $\mathfrak{g}_{1}^{ \pm \alpha}=K e_{ \pm \alpha}$ and $h_{\alpha}=\left[e_{\alpha}, e_{-\alpha}\right]$.

1.6. We construct a functor $T$ between categories of left modules:

$$
T: U\left(\mathfrak{g}_{0}\right)-\bmod \longrightarrow U(\mathfrak{g}) \text {-mod. }
$$

First set $\mathfrak{p}=\mathfrak{g}_{0} \oplus \mathfrak{g}_{1}^{+}$and $J=U(\mathfrak{p}) \mathfrak{g}_{1}^{+}$. Then $J$ is a nilpotent ideal of $U(\mathfrak{p})$ with $U(\mathfrak{p}) / J \cong U\left(\mathfrak{g}_{0}\right)$. Thus we can regard $U\left(\mathfrak{g}_{0}\right)$-mod as a subcategory of $U(\mathfrak{p})$-mod and define $T\left({ }_{-}\right)=U(\mathfrak{g}) \otimes_{U(\mathfrak{p})}\left({ }_{-}\right)$.

We use the functor $T$ to construct some examples of P.I. envelopes of $\mathfrak{g}$. If $M$ is any $K[z]$-module and $\lambda \in P^{+}$we regard $L(\lambda, M)=L_{\lambda} \otimes M$ as a $U\left(\mathfrak{g}_{0}\right)$-module by allowing $\left[\mathfrak{g}_{0}, \mathfrak{g}_{0}\right]$ (resp. $\left.K z\right)$ to act on the first (resp. second) factor of the tensor product. For $a \in K$, let $\mathcal{O}_{a}=K[z] /(z-a)$ and set

$$
\begin{aligned}
& L(\lambda)=L(\lambda, K[z]), L(\lambda, a)=L\left(\lambda, \mathcal{O}_{a}\right), \\
& V(\lambda)=T(L(\lambda)), V(\lambda, a)=T(L(\lambda, a)) .
\end{aligned}
$$

The natural map $K[z] \longrightarrow \mathcal{O}_{a}$ induces an epimorphism of $U(\mathfrak{g})$-modules

$$
V(\lambda) \longrightarrow V(\lambda, a) .
$$

The pair $\lambda^{\prime}=(\lambda, a)$ can be viewed as the element of $(\mathfrak{h})^{*}$ with $\left.\lambda^{\prime}\right|_{\mathfrak{h}}=\lambda$ and $\lambda(z)=a$. The module $V(\lambda, a)$ is called the Kac module with highest weight $\lambda^{\prime}$. By [K2, Proposition 2.9] $V(\lambda, a)$ is a simple $U(\mathfrak{g})$-module if and only if $\lambda^{\prime}=(\lambda, a)$ satisfies $\left(\lambda^{\prime}+\rho, \alpha\right) \neq 0$ for all odd positive roots $\alpha$. Here $\rho=\rho_{0}-\rho_{1}$, where $\rho_{0}$ (resp. $\rho_{1}$ ) is the half-sum of the positive even (resp. odd) roots. Since $V(\lambda)$ maps onto any Kac module of the form $V(\lambda, a)$ we call $V(\lambda)$ the universal Kac-module with highest weight $\lambda \in \mathfrak{h}^{*}$. 
1.7. The enveloping algebra $U$ has a $\mathbb{Z}$-grading, $U=\bigoplus_{n \in \mathbb{Z}} U(n)$ extending the $\mathbb{Z}$ grading on $\mathfrak{g}$ given by $\operatorname{deg} \mathfrak{g}_{0}=0, \operatorname{deg} \mathfrak{g}_{1}^{ \pm}= \pm 1$. Henceforth the adjective "graded" refers to this grading. We use this grading to construct a useful localization of $U$.

Suppose that $M=\bigoplus M(n)$ is a graded $U$-module which is torsionfree as a $K[z]$-module. We can make $M$ into a $U-K[z]$-bimodule via the rule

$$
m f(z)=f(z-n) m
$$

for $m \in M(n), f(z) \in K[z]$. Let $F=K(z)$ and give $M^{F}=M \otimes_{K[z]} F$ the right $F$-module structure obtained by localization. In particular $U^{F}$ becomes a $U-F$ bimodule in this way and we can extend the algebra structure on $U$ to $U^{F}$ by

$$
\left(u \otimes f_{1}(z)\right)\left(v \otimes f_{2}(z)\right)=u v \otimes f_{1}(z+n) f_{2}(z)
$$

for $u \in U, v \in U(n)$ and $f_{1}, f_{2} \in F$. It is now easy to verify the following:

Lemma. The multiplicative set $\mathcal{C}=K[z] \backslash\{0\}$ is Ore in $U$ and $U_{\mathcal{C}} \cong U^{F}$ with the above algebra structure. If $M$ is a graded left $U$-module which is torsionfree as a $K[z]$-module, then $M_{\mathcal{C}} \cong M \otimes_{K[z]} F$ as a $U-F$ bimodule via the map

$$
f^{-1}(z) m \longrightarrow m \otimes f^{-1}(z+n)
$$

for $m \in M(n), f(z) \in K[z]$. In addition if $N$ is any graded $U$-submodule of $M_{\mathcal{C}}$, then $N$ is a $U_{\mathcal{C}}$-submodule if any only if it is a $U-F$ sub-bimodule of $M_{\mathcal{C}}$.

1.8. If $V$ is a vector space over $K$ we write $V_{F}$ for $V \otimes F$. If $A$ is a $K$-algebra and $M$ is a left $A$-module, then $A_{F}$ is an $F$-algebra and $M_{F}$ an $A_{F}$-module by extension of scalars.

We apply these remarks to the universal Kac module $V(\lambda)=U(\mathfrak{g}) \otimes_{U(\mathfrak{p})} L(\lambda)$. If $\Lambda=\bigoplus \Lambda^{n}$ is the exterior algebra on $\mathfrak{g}_{1}^{-}$, then as a left $U\left(\mathfrak{g}_{0}\right)$-module

$$
V(\lambda) \cong \bigoplus \Lambda^{n} \otimes L(\lambda) \text {. }
$$

By definition $L(\lambda)=L_{\lambda} \otimes K[z]$, and so $L(\lambda)$ and $V(\lambda)$ are in an obvious way right $K[z]$-modules. Since $\operatorname{deg} \mathfrak{g}_{1}^{-}=-1$ the gradings on $\Lambda$ and $U$ satisfy $\Lambda^{n} \subseteq U(-n)$. Observe that the $K[z]$-bimodule structure on $V(\lambda)$ satisfies (1) in section 1.7.

Note also that $V(\lambda)$ is torsionfree as a left (and right) $K[z]$-module. Thus $V(\lambda)_{\mathcal{C}}$ is a $U-F$ bimodule or equivalently a left $U_{F}$-module and we have

$$
\begin{aligned}
V(\lambda)_{\mathcal{C}} & \cong\left(U(\mathfrak{g}) \otimes_{U(\mathfrak{p})} L(\lambda)\right) \otimes_{K[z]} F \\
& \cong U(\mathfrak{g}) \otimes_{U(\mathfrak{p})}\left(L(\lambda) \otimes_{K[z]} F\right) \\
& \cong U(\mathfrak{g})_{F} \otimes_{U(\mathfrak{p})_{F}}\left(L(\lambda) \otimes_{K[z]} F\right) .
\end{aligned}
$$

Next we consider the $U(\mathfrak{p})_{F}$-module $L(\lambda) \otimes F$. This is annihilated by $\mathfrak{g}_{1 F}^{+}$and so is a $U\left(\mathfrak{g}_{0}\right)_{F}$-module. In fact it is the finite dimensional simple module over this algebra whose highest weight $\lambda^{\prime}$ is the unique $F$-linear map $\mathfrak{h}_{F}^{\prime} \longrightarrow F$ such that $\left.\lambda^{\prime}\right|_{\mathfrak{h}}=\lambda$ and $\lambda^{\prime}(z)=z$. Thus $V(\lambda)_{\mathcal{C}}$ is a Kac module over $U(\mathfrak{g}) \otimes F$. In fact we have

Proposition. (a) The module $V(\lambda)_{\mathcal{C}}$ is a simple Kac module with highest weight $\lambda^{\prime}$ module over the algebra $U(\mathfrak{g}) \otimes F$.

(b) The module $V(\lambda)_{\mathcal{C}}$ is a simple module over the algebra $U(\mathfrak{g})_{\mathcal{C}}$.

Proof. It remains to show simplicity in both cases.

(a) We extend $($,$) to a bilinear form (,)_{F}$ on $\left(\mathfrak{h}^{\prime}\right)_{F}^{*}$. It suffices to show that $\left(\lambda^{\prime}+\rho, \alpha\right) \neq 0$ for all odd positive roots $\alpha$. Since the highest exterior power of $\mathfrak{g}_{1}^{+}$ 
is trivial as a $\mathfrak{g}_{0}$-module we have $\left(\rho_{1}, \beta\right)=0$ for all even roots $\beta$. Thus $\mathfrak{h}^{\perp}=K \rho_{1}$. If $\alpha$ is an odd root, it follows that $\left(\rho_{1}, \alpha\right)=\rho_{1}\left(h_{\alpha}\right) \neq 0$. Hence $h_{\alpha} \notin\left(K \rho_{1}\right)^{\perp}=\mathfrak{h}$, so $h_{\alpha}-b z \in \mathfrak{h}$ for some nonzero $b \in K$. Therefore

$$
\left(\lambda^{\prime}+\rho, \alpha\right)_{F}=\lambda^{\prime}\left(h_{\alpha}-b z+b z\right)_{F}+(\rho, \alpha)=b z+\lambda\left(h_{\alpha}-b z\right)+(\rho, \alpha),
$$

which is a linear polynomial in $z$.

(b) Fix an order on $\Delta_{1}^{+}$and for $I \subseteq \Delta_{1}^{+}$set

$$
e_{I}=\prod_{\alpha \in I} e_{-\alpha}
$$

where the product is taken with respect to this order. Let $N$ be a nonzero $U(\mathfrak{g})_{\mathcal{C}^{-}}$ submodule of $V(\lambda)_{\mathcal{C}}$ and suppose

$$
n=\sum_{I} e_{I} n_{I}
$$

is nonzero with $n_{I} \in L(\lambda) \otimes_{K[z]} F$ for all $I$. Choose $m$ minimal such that $n_{I} \neq 0$ for some subset $I$ with $|I|=m$. Set $I^{\prime}=\Delta_{1}^{+} \backslash I$. Then

$$
e_{I^{\prime}} n= \pm e_{\Delta_{1}^{+}} n_{I}
$$

is a nonzero homogeneous element of $N$, so generates a graded submodule. It follows from Lemma 1.7 that $N=V(\lambda)_{\mathcal{C}}$.

Corollary. (a) If $N$ is any nonzero $U(\mathfrak{g})$-submodule of $V(\lambda)$, then $N$ contains a submodule isomorphic to $V(\lambda)$.

(b) $P_{\lambda}=\operatorname{ann}_{U(\mathfrak{g})} V(\lambda)$ is a prime ideal of $U(\mathfrak{g})$.

Proof. (a) By the Proposition $N_{\mathcal{C}}=(V(\lambda))_{\mathcal{C}}$. Hence $\left(L_{\lambda} \otimes 1\right) \subseteq N_{\mathcal{C}}$ so $L_{\lambda} \otimes(f) \subseteq N$ for some nonzero $f$. The submodule of $V(\lambda)$ generated by $L_{\lambda} \otimes(f)$ is isomorphic to $T(L(\lambda,(f))) \cong V(\lambda)$.

(b) This follows since any nonzero submodule of $V(\lambda)$ has annihilator $P_{\lambda}$, by part (a).

1.9. Lemma. Identify $L(\lambda)$ with the $U(\mathfrak{p})$-submodule $1 \otimes L(\lambda)$ of $V(\lambda)$ and let $J=U(\mathfrak{p}) \mathfrak{g}_{1}^{+}$. Then ann $_{V(\lambda)} J=L(\lambda)$.

Proof. We use the same notation as in the proof of Proposition 1.8(b).

For $0 \leq m \leq\left|\Delta_{1}^{+}\right|$set

$$
V(m)=\bigoplus_{|I|=m} e_{I} L(\lambda)
$$

Then $V(\lambda)=\bigoplus_{m} V(m)$ and $\mathfrak{g}_{1}^{+} V(m) \subseteq V(m-1)$. Therefore it suffices to show that if $m>0$, then $\mathfrak{g}_{1}^{+}\left(\sum_{|I|=m} e_{I} w_{I}\right) \neq 0$ provided the $w_{I} \in L(\lambda)$ are not all zero. Now $L(\lambda)=L_{\lambda} \otimes K[z]$ has a filtration given by setting $\operatorname{deg}\left(L_{\lambda} \otimes z^{n}\right)=n$. Choose $I$ so that $w_{I}$ has maximum degree in this filtration, choose $\alpha \in I$ and set $H=I \backslash\{\alpha\}$. Note that

Using the formula

$$
\operatorname{deg}\left(h_{\alpha} w_{I}\right)=\operatorname{deg}\left(w_{I}\right)+1 .
$$

$$
\left[e_{\alpha}, a b\right]=\left[e_{\alpha}, a\right] b \pm a\left[e_{\alpha}, b\right]
$$

for homogeneous $a, b \in U(\mathfrak{g})$ we see that

$$
e_{\alpha} e_{I} w_{I}= \pm e_{H} h_{\alpha} w_{I}
$$

plus a sum of terms of smaller degree. The result follows easily from this. 
1.10. The next result is an easy consequence of the Artin-Wedderburn theorem.

Lemma. Let $U$ be a $K$-algebra and $L$ a finite dimensional simple $U$-module. Then for any field extension $K^{\prime}$ of $K$ we have

$$
\operatorname{End}_{U \otimes_{K} K^{\prime}}\left(L \otimes_{K} K^{\prime}\right)=K^{\prime} .
$$

1.11. Lemma. For any $\lambda \in P^{+}$,

$$
\operatorname{End}_{U(\mathfrak{g})}(V(\lambda)) \cong K[z] .
$$

Proof. By the adjoint isomorphism $f \in \operatorname{End}_{U(\mathfrak{g})}(V(\lambda))$ is determined by

$$
f_{1}=\left.f\right|_{L(\lambda)} \in \operatorname{Hom}_{U(\mathfrak{p})}(L(\lambda), V(\lambda)) .
$$

If $J$ is as in Lemma 1.9, then $f_{1}(L(\lambda)) \subseteq a n n_{V(\lambda)} J=L(\lambda)$, and hence

$$
f_{1} \in \operatorname{End}_{U(\mathfrak{p})}\left(L_{\lambda} \otimes K[z]\right)=\operatorname{End}_{U\left(\mathfrak{g}_{0}\right)}\left(L_{\lambda} \otimes K[z]\right) \cong K[z],
$$

using Lemma 1.10.

1.12. Suppose that $\mathfrak{k}$ is a semisimple Lie algebra over $K$ and $C$ is a commutative $K$-algebra. We describe the prime ideals $Q$ of $R=U(\mathfrak{k}) \otimes C$ such that $R / Q$ is P.I. Since $C$ is central, $q=Q \cap C$ is prime in $C$ and by replacing $R$ by the factor ring $R / R q$ we can assume that $Q \cap C=0$. There is a one-one correspondence between prime ideals $Q$ of $R$ such that $Q \cap C=0$ and prime ideals of $U(\mathfrak{k}) \otimes \operatorname{Fract}(C)$. Thus we may assume that $C$ is a field extension of $K$. By the argument on page 2837 of [BM] $Q$ is the annihilator of a finite dimensional simple module over $U(\mathfrak{k}) \otimes C$.

To apply this to our reductive algebra $\mathfrak{g}_{0}$ with center $K z$ set $\mathfrak{k}=\left[\mathfrak{g}_{0}, \mathfrak{g}_{0}\right], R=$ $U\left(\mathfrak{g}_{0}\right)$ and $C=K[z]$. If $q \neq 0$, then $R / R q \cong U(\mathfrak{k})$ and $R / Q$ is artinian. Thus if $R / Q$ is nonartinian, then $q=0$ and $Q$ corresponds to the annihilator of a finite dimensional simple module over $U(\mathfrak{k}) \otimes \operatorname{Fract}(C)$. This gives the following result.

Lemma. Suppose $Q$ is a prime ideal of $U\left(\mathfrak{g}_{0}\right)$ such that $U\left(\mathfrak{g}_{0}\right) / Q$ is a nonartinian P.I. ring. Then for some uniquely determined $\lambda \in P^{+}, Q=$ ann $_{U\left(\mathfrak{g}_{0}\right)} L(\lambda)$.

Proof of the Main Theorem. Suppose $\lambda \in P^{+}$and let $n=\operatorname{dim}_{K} L_{\lambda}$. By Corollary $1.8 P_{\lambda}$ is a prime ideal in $U(\mathfrak{g})$. Set $U_{\lambda}=U(\mathfrak{g}) / P_{\lambda}$. Note that $V(\lambda)$ is a torsionfree $K[z]$-module and thus $U_{\lambda}$ embeds in $\left(U_{\lambda}\right)_{\mathcal{C}}$. Since $V(\lambda)$ is a $U(\mathfrak{g})-K[z]$-bimodule which is free of rank $N=n 2^{\operatorname{dim} \mathfrak{g}_{1}^{-}}$on the right, $U_{\lambda}$ embeds in $M_{N}(K[z])$. This embedding induces an embedding of $\left(U_{\lambda}\right)_{\mathcal{C}}$ into $M_{N}(F)$ which is surjective since $V(\lambda)_{\mathcal{C}}$ is a simple $\left(U_{\lambda}\right)_{\mathcal{C}}$-module of dimension $N$ over its endomorphism $\operatorname{ring} F$.

Conversely suppose $P$ is a prime ideal of $U(\mathfrak{g})$ with $U(\mathfrak{g}) / P$ a nonartinian P.I. ring. We apply the results in sections 1.1 and 1.2 with $R=U(\mathfrak{p})$ and $S=U(\mathfrak{g})$. If $Q \in V_{P}$ there is a bond from $S / P$ to $R / Q$, so $R / Q$ is a nonartinian P.I. ring, by Lemma 1.1. Lemma 1.12 implies that $Q=Q_{\lambda}$ for some $\lambda \in P^{+}$. Since $P \in X_{Q}, P$ is minimal over $\operatorname{ann}_{S}(S / S Q)$ which equals $\operatorname{ann}_{S} V(\lambda)=P_{\lambda}$ by [BGR, Satz 10.4]. As $P_{\lambda}$ is prime we get $P=P_{\lambda}$. To show that $\lambda$ is uniquely determined by $P$ it suffices to show that $V_{P_{\lambda}}=\left\{Q_{\lambda}\right\}$. However if $Q^{\prime} \in V_{P_{\lambda}}$, then by Lemma $1.4 Q^{\prime}=a_{n} n_{R} N$ for some $R$-submodule $N$ of $V(\lambda)$ which is fully faithful as an $R / Q^{\prime}$-submodule. Since $J=U(\mathfrak{p}) \mathfrak{g}_{1}^{+}$is nilpotent $J \subseteq Q^{\prime}$, so using Lemma $1.9 N \subseteq \operatorname{ann}_{V(\lambda)} J=L(\lambda)$. However every nonzero submodule of $L(\lambda)$ has annihilator $\bar{Q}_{\lambda}$, so $Q^{\prime}=Q_{\lambda}$ as desired. 


\section{REFERENCES}

[BM] Y. Bahturin and S. Montgomery, PI-envelopes of Lie superalgebras, Proc. Amer. Math. Soc., 127 (1999), 2829-2839. MR 2000a:17029

[BGR] W. Borho, P. Gabriel and R. Rentschler, Primideale in Einhüllenden aufösbarer LieAlgebren, Lecture notes in Mathematics, 357, Springer-Verlag, Berlin, 1973. MR 51:12965

[BS] K.A. Brown and S.P. Smith, Bimodules over a solvable algebraic Lie algebra, Quart. J. Math. 36 (1985), 129-139. MR 87d:17009

[J] A.V. Jategaonkar, Localization in Noetherian Rings, LMS Lecture Notes 98, Cambridge University Press, Cambridge 1986. MR 88c:16005

[K1] V.G. Kac, Lie Superalgebras, Adv. in Math., 26 (1977), 8-96. MR 58:5803

[K2] V.G. Kac, Representations of classical Lie superalgebras, pages 597-626. Differential Geometric Methods in Mathematical Physics II, ed. K. Bleuler et al. Lecture Notes in Mathematics, 676, Springer-Verlag, Berlin, 1978. MR 80f:17006

[L1] E.S. Letzter, Primitive ideals in finite extensions of Noetherian rings, J. London Math. Soc. 39 (1989), 427-435. MR 90f:16013

[L2] E.S. Letzter, Finite correspondence of spectra in Noetherian ring extensions, Proc. Amer. Math. Soc. 116 (1992), 645-652. MR 93a:16003

[L3] E.S. Letzter, A bijection of spectra for classical Lie superalgebras of Type I, J. London Math. Soc. 53 (1996), 39-49. MR 96k:17016

[McR] J.C. McConnell and J.C. Robson, Noncommutative Noetherian rings, Wiley-Interscience, Chichester 1987. MR 89j:16023 revised edition MR 2001i:16039

Department of Mathematical Sciences, University of Wisconsin, Milwaukee, WisconSIN 53211

E-mail address: musson@csd.uwm.edu 